\title{
Boas Escolhas e Fatores de Risco Associados ao Envelhecimento Saudável: Revisão da Literatura
}

\author{
Good Choices and Risk Factors Associated with Healthy \\ Aging: Literature Review
}

\begin{abstract}
BATISTA, C.C. ${ }^{1}$; OLIVEIRA, OD²; MARRONE, L.C.P. ${ }^{3} ;$ MORGAN-MARTINS, M.I. ${ }^{4 *}$

${ }^{1}$ Aluno do Programa de Mestrado em Promoção da Saúde Desenvolvimento Humano e Sociedade- Universidade Luterana do Brasil (ULBRA)- Canoas- RS ${ }^{2}$ Aluna do Programa de Mestrado em Promoção da Saúde Desenvolvimento Humano e Sociedade- Universidade Luterana do Brasil (ULBRA)- Canoas- RS
\end{abstract}

3Professor Adjunto do Programa de Mestrado em Promoção da Saúde Desenvolvimento Humano e Sociedade- Universidade Luterana do Brasil (ULBRA) - Canoas- RS

4Professora Adjunto do Programa de Mestrado em Promoção da Saúde Desenvolvimento Humano e Sociedade- Universidade Luterana do Brasil (ULBRA) - Canoas- RS

*Autor correspondente- E-mail: mimorganm@gmail.com

DOI: https://doi.org/10.53817/1983-6929-ragg-v12n1-1

\section{RESUMO}

Introdução: No mundo vem ocorrendo uma inversão da pirâmide etária da população mundial. Há uma redução expressiva nas taxas de natalidade associada a uma elevação da expectativa de vida causando uma modificação no percentual de idosos. Muito se deve ao avanço tecnológico na saúde e nas condições gerais, bem como, a bons hábitos de vida. Objetivo: Assim, o objetivo desse estudo foi discutir o processo do envelhecimento saudável e algumas alterações inerentes a esse. Metodologia: A revisão da literatura foi realizada no período de 2000 a 2019, nas bases de dados da BVS (Biblioteca Virtual em Saúde) e PubMed (US National Library of Medicine/National Institutes of Health). Resultados: Foram selecionados 38 artigos na BVS e 44 no PubMed, totalizando 92. Desses 22 foram considerados de maior relevância para esse artigo. Discussão: $O$ envelhecimento traz à tona a fragilidade da condição humana encarnando-se o "irredutível do corpo" por não ser mais capaz de participar do campo simbólico da sociedade. Porém, a longevidade inicia a partir de boas escolhas que desencadeiam bons hábitos, como a prática de atividade física e ser criativo. As oportunidades e experiências devem estar associados a promoção da saúde integral do idoso, oportunidades de estudo, de trabalho para 
quem gosta e precisa, acesso a saúde física e mental, acesso ao laser, acesso a moradia digna e vida em comunidade. Conclusão: Portanto, o envelhecimento ativo, como preconiza a ONU, deve estar associado as boas escolhas ao longo da vida e isso irá proporcionar novas oportunidades para viverem novas experiências com qualidade de vida.

Palavras chaves: "longevidade", "envelhecimento saudável”, "promoção da saúde".

\section{ABSTRACT}

Introduction: There is an inversion of the world population age pyramid in the world. There is a significant reduction in birth rates associated with an increase in life expectancy causing a change in the percentage of elderly people. Much is due to technological advances in health and general conditions, as well as good lifestyle habits. Objective: Thus, the objective of this study was to discuss the healthy aging process and some changes inherent to it. Methodology: The literature review was carried out from 2000 to 2019, in the databases of the VHL (Virtual Health Library) and PubMed (US National Library of Medicine/National Institutes of Health). Results: 38 articles were selected from the VHL and 44 from PubMed, totaling 92. Of these, 22 were considered of greater relevance for this article. Discussion: Aging brings out the fragility of the human condition, embodying the "irreducible of the body" for no longer being able to participate in the symbolic field of society. However, longevity starts with good choices that trigger good habits, such as physical activity and being creative. Opportunities and experiences must be associated with the promotion of integral health for the elderly, opportunities for study, work for those who like and need it, access to physical and mental health, access to laser, access to decent housing and community life. Conclusion: Therefore, active aging, as recommended by the UN, must be associated with good choices throughout life and this will provide new opportunities to live new experiences with quality of life.Keywords: Iongevity, healthy aging, health promotion.

\section{Introdução}

No mundo, há um aumento da população idosa acima dos 65 anos, sendo que a projeção de crescimento da população mundial, que em 2019 foi de 7,7 bilhões para 2030 é estimado 8,5 bilhões, havendo um aumento de $10 \%$ neste crescimento. Em contrapartida a taxa de crescimento varia muito em cada região, o que está desencadeando uma inversão na pirâmide etária da população mundial. É possível que este aumento da população idosa se deva um aumento da expectativa de vida associado há uma redução nas taxas de fertilidade (WPP, 2019).

O envelhecimento da população altera inúmeros fatores na sociedade, como as questões relacionadas à saúde, economia e aspectos sociais. Em função disso, torna-se fundamental a discussão de aspectos relacionados à saúde e a qualidade de vida do idoso, bem como, conhecer o perfil dos idosos de diferentes regiões, a fim de promover ações promotoras de saúde (WHO, 2012; LLOYD-SHERLOCK P, MCKEE M, EBRAHIM S, et al., 2012). 
Por isso, o envelhecimento da população nunca será um fenômeno isolado, mas, está associado a modificações do perfil epidemiológico e das características sociais e econômicas das populações. É visível uma transição demográfica no tamanho e na estrutura etária da população, e que acompanha a evolução socioeconômica presente na maioria dos países que nem sempre é um aspecto positivo (GIACOMIN; SANTOS; FIRMO, 2013).

Portanto, o envelhecimento é definido como a última fase do ciclo vital e é delimitado por eventos de natureza múltipla, incluindo, por exemplo, perdas psicomotoras, afastamento social, restrição em papéis sociais e redução cognitiva. Portanto, o processo fisiológico do envelhecimento está diretamente associado as transformações pelo qual o organismo vai sofrendo ao longo da vida, resultando na diminuição gradual da expectativa de sobrevivência (FREITAS; QUEIROZ; SOUSA, 2010).

Assim, a Organização Mundial da Saúde (OMS) afirma que envelhecer deveria estar diretamente associado com saúde e ser preocupação de todo ser humano ao longo da vida. Contudo, quando o envelhecimento chega traz consigo uma carga resultante dos hábitos adquiridos ao longo da vida, que por vezes está associado a maus hábitos alimentares, consumo de bebida alcóolica, uso de tabaco e o sedentarismo (OMS, 2005). Portanto, envelhecer deve estar associado ao envelhecimento ativo e produtivo que proporcionará novas experiências de vida. Estas devem estar associados a promoção da saúde, permitindo que o indivíduo possa ser capaz de continuar contribuindo com a sociedade, mantendo um estilo de vida ativo em que ele possa fazer escolhas e adquirir novos conhecimentos (BRYANT; ALPETER; WHITELAW, 2006).

Muitos problemas de saúde da fase adulta e da velhice são resultados dos hábitos e do estilo de vida adquiridos ao longo da vida. Os hábitos alimentares, a ingestão de álcool em excesso, o hábito de fumar, o sedentarismo e outros fatores trazem uma série de consequências na vida do ser humano principalmente aqueles associados as doenças crônicas, como: diabetes, hipertensão, acidentes vasculares, doenças degenerativas e o câncer. Nesse sentido a possibilidade de viver de forma mais longeva e com boa saúde é maior se a pessoa optar por hábitos que proporcionem um estilo de vida saudável (BAUMAN, 2011).

Portanto, o envelhecimento não é considerado uma doença, mas uma fase do ciclo vital que seria desejável estar associado a: independência, autonomia, autoeficácia, autoestima, otimismo, bom humor, harmonia intergeracional, com capital social e financeiro. Portanto, para promover saúde no envelhecimento há a necessidade de promover ações de melhoria e manutenção da qualidade de vida e que estejam relacionadas as escolhas e aos bons hábitos ao longo da vida. Assim, o estudo tem como objetivo discutir o processo do envelhecimento saudável e algumas alterações inerentes a esse.

\section{Metodologia}

Esse é um estudo descritivo, exploratório que utilizou uma abordagem qualitativa de revisão da literatura. Para a elaboração do artigo foram utilizados os seguintes descritores: "Longevity", "healthy aging" and "health promotion". A 
busca foi realizada no período de Julho de 2019, utilizando como filtro o período de inclusão 2000 a 2019, nas bases de dados da BVS (Biblioteca Virtual em Saúde) e PubMed (US National Library of Medicine/National Institutes of Health.

Após a leitura e análise dos artigos, foram selecionados 38 artigos na plataforma BVS e 44 na plataforma PubMed, totalizando 92 artigos. Para que fosse possível selecionar os artigos pertinentes ao tema, foi realizado a leitura dos títulos e desconsiderados aqueles com títulos repetidos, sendo excluídos 12 artigos nesta fase. Logo após, realizado uma leitura minuciosa dos resumos, para exclusão de artigos que não se adequassem a temática, sendo 28 artigos excluídos. Após essa fase, foi realizada a leitura do texto dos artigos, na integra, e selecionados aqueles cujo conteúdo fosse relacionado a envelhecimento ativo (saudável), longevidade e promoção da saúde, sendo excluídos 30 artigos nesta fase. Ao final das leituras, 22 artigos se adequaram à temática e compõe as referências da presente revisão.

Esta pesquisa está vinculada ao projeto "Longevidade e os hábitos de vida dos idosos do município de Itaituba, Pará", com parecer do comitê de ética da Universidade Luterana do Brasil (ULBRA - Canoas/RS) - Comunidade Evangélica, sendo aprovado conforme parecer número 3.423.392, CAAE: 11843619.0.0000.5349.

\section{Resultados}

A partir das leituras, foram extraídas cinco temáticas que mais se repetiram nos textos, sendo elas: envelhecimento; fatores que aceleram o processo de envelhecimento; fatores que retardam 0 processo de envelhecimento; longevidade e estilo de vida; e promoção da saúde. O quadro abaixo apresenta a relação das publicações encontradas.

Tabela: Relação das publicações encontradas nas bases de dados BVS (Biblioteca Virtual em Saúde) e PubMed (US National Library of Medicine/National Institutes of Health.

\begin{tabular}{|c|c|c|c|c|c|}
\hline & Autor & Ano & Título & Periódico & Tipo \\
\hline 1 & $\begin{array}{lr}\text { BRYANT } & \text { L, } \\
\text { ALPETER } & M, \\
\text { WHITELAW N. } & \end{array}$ & 2006 & $\begin{array}{l}\text { Evaluation of Health } \\
\text { PromotionPrograms for Older } \\
\text { Adults:An Introduction. }\end{array}$ & $\begin{array}{l}\text { Journal of Applied } \\
\text { Gerontology. }\end{array}$ & $\begin{array}{l}\text { Revisão de } \\
\text { literatura }\end{array}$ \\
\hline 2 & $\begin{array}{l}\text { CAMPOS M, NETO } \\
\text { J. }\end{array}$ & 2008 & $\begin{array}{l}\text { Qualidade de vida: um } \\
\text { instrumento para promoção da } \\
\text { saúde }\end{array}$ & $\begin{array}{l}\text { Revista Baiana de } \\
\text { Saúde Pública }\end{array}$ & $\begin{array}{l}\text { Revisão de } \\
\text { literatura }\end{array}$ \\
\hline 3 & COSTA, JJS & 2015 & $\begin{array}{l}\text { A educação segundo Paulo } \\
\text { Freire: uma primeira análise } \\
\text { filosófica. }\end{array}$ & $\begin{array}{l}\text { Theoria - Revista } \\
\text { Eletrônica de Filosofia } \\
\text { Faculdade Católica de } \\
\text { Porto Alegre. }\end{array}$ & $\begin{array}{l}\text { Revisão de } \\
\text { literatura }\end{array}$ \\
\hline 4 & FERNÁNDEZ, BR. & 2009 & $\begin{array}{l}\text { Envejececimiento activo. } \\
\text { Contribuciones de la } \\
\text { Psicología. }\end{array}$ & RIDEP & $\begin{array}{l}\text { Artigo de } \\
\text { Revisão de } \\
\text { literatura }\end{array}$ \\
\hline 5 & FERREIRA, A. & 2009 & $\begin{array}{l}\text { A Qualidade de Vida em } \\
\text { Idosos em Diferentes } \\
\text { Contextos Habitacionais: A }\end{array}$ & $\begin{array}{l}\text { Faculdade de } \\
\text { Psicologia e Ciências } \\
\text { da Educação, } \\
\text { Universidade de Lisboa }\end{array}$ & $\begin{array}{l}\text { Dissertação de } \\
\text { Mestrado }\end{array}$ \\
\hline
\end{tabular}




\begin{tabular}{|c|c|c|c|c|c|}
\hline & & & $\begin{array}{l}\text { Perspectiva do Próprio e do } \\
\text { Seu Cuidador. }\end{array}$ & & \\
\hline 6 & FONSECA A. & 2006 & $\begin{array}{l}\text { O envelhecimento. Uma } \\
\text { abordagem psicológica. }\end{array}$ & $\begin{array}{l}\text { Universidade Católica } \\
\text { Editora }\end{array}$ & Livro \\
\hline 7 & $\begin{array}{lc}\text { FREITAS } & \mathrm{M}, \\
\text { QUEIROZ } & \mathrm{T}, \\
\text { SOUSA J } & \end{array}$ & 2010 & $\begin{array}{l}\text { O significado da velhice e da } \\
\text { experiência de envelhecer para } \\
\text { os idosos. }\end{array}$ & $\begin{array}{l}\text { Revista da Escola de } \\
\text { Enfermagem da } \\
\text { Universidade de São } \\
\text { Paulo }\end{array}$ & $\begin{array}{l}\text { Pesquisa } \\
\text { exploratória, } \\
\text { descritiva, } \\
\text { qualitativa. }\end{array}$ \\
\hline 8 & $\begin{array}{ll}\text { FRIES } & \text { AT, } \\
\text { PEREIRA DC. } & \end{array}$ & 2011 & Teorias do envelhecimento. & $\begin{array}{l}\text { Revista Contexto \& } \\
\text { Saúde }\end{array}$ & $\begin{array}{l}\text { Revisão de } \\
\text { literatura. }\end{array}$ \\
\hline 9 & $\begin{array}{ll}\text { GIACOMIN } & \mathrm{KC}, \\
\text { SANTOS } & \text { WJ, } \\
\text { FIRMO JOA. } & \end{array}$ & 2013 & $\begin{array}{l}\text { O luto antecipado diante da } \\
\text { consciência da finitude: a vida } \\
\text { entre os medos de não dar } \\
\text { conta, de dar trabalho e de } \\
\text { morrer. }\end{array}$ & Ciênc. Saúde Coletiva & $\begin{array}{l}\text { Estudo } \\
\text { qualitativo. }\end{array}$ \\
\hline 10 & $\begin{array}{ll}\text { IRIGARAY } & \text { T, } \\
\text { TRENTINI C. } & \end{array}$ & 2009 & $\begin{array}{l}\text { Qualidade de vida em idosas: a } \\
\text { importância da dimensão } \\
\text { subjetiva. }\end{array}$ & Estudos de Psicologia & $\begin{array}{l}\text { Quantitativo/ } \\
\text { transversal }\end{array}$ \\
\hline 11 & KALACHE A. & 2008 & $\begin{array}{l}\text { O mundo envelhece: é } \\
\text { imperativo criar um pacto de } \\
\text { solidariedade }\end{array}$ & $\begin{array}{l}\text { Social Ciência \& Saúde } \\
\text { Coletiva }\end{array}$ & $\begin{array}{l}\text { Revisão de } \\
\text { literatura }\end{array}$ \\
\hline 12 & LE BRETON D. & 2012 & Sociologia do corpo. & $\begin{array}{l}\text { Editora Vozes / São } \\
\text { Paulo }\end{array}$ & Livro \\
\hline 13 & $\begin{array}{ll}\text { MINAYO } & \text { MCS, } \\
\text { ASSIS SG } & \end{array}$ & 2017 & $\begin{array}{l}\text { Novas e velhas faces da } \\
\text { violência no Século XXI: Visão } \\
\text { da literatura brasileira do } \\
\text { campo da saúde }\end{array}$ & $\begin{array}{l}\text { Editora Fiocruz / Rio de } \\
\text { Janeiro }\end{array}$ & Livro \\
\hline 14 & $\begin{array}{ll}\text { MARRONI } & \text { NP, } \\
\text { MORGAN- } & \\
\text { MARTINS } & \text { MI; } \\
\text { PORAWASKI } & \end{array}$ & 2012 & $\begin{array}{l}\text { Radicais livres no processo } \\
\text { saúde-doença: da bancada à } \\
\text { clínica. }\end{array}$ & Editora CRV / Curitiba & Livro \\
\hline 15 & NUNES, IM. & 2009 & $\begin{array}{l}\text { A Constelação do Idoso. Artigo } \\
\text { de Investigação Médica. }\end{array}$ & $\begin{array}{l}\text { Instituto de Ciências } \\
\text { Biomédicas Abel } \\
\text { Salazar }\end{array}$ & $\begin{array}{l}\text { Tese de } \\
\text { mestrado }\end{array}$ \\
\hline 16 & $\begin{array}{l}\text { PEREIRA ÉF, } \\
\text { TEIXEIRA CS, } \\
\text { DARONCO LSE }\end{array}$ & 2009 & $\begin{array}{l}\text { Estilo de vida, prática de } \\
\text { exercício físico e dores } \\
\text { musculoesqueléticas em } \\
\text { idosas fisicamente ativas. }\end{array}$ & RBCEH, Passo Fundo & $\begin{array}{l}\text { Quantitativo/ } \\
\text { transversal }\end{array}$ \\
\hline 17 & $\begin{array}{l}\text { PORCELLO- } \\
\text { MARRONE LC et al }\end{array}$ & 2013 & $\begin{array}{l}\text { Risk factors among stroke } \\
\text { subtypes in Brazil. }\end{array}$ & $\begin{array}{l}\text { Journal of Stroke and } \\
\text { Cerebrovascular } \\
\text { Diseases }\end{array}$ & Quantitativo \\
\hline 18 & RESENDE, MR PL. & 2009 & $\begin{array}{l}\text { Imunocosmética estimulação } \\
\text { de células imunocompetentes } \\
\text { para a proteção da pele e } \\
\text { reparação do DNA. }\end{array}$ & $\begin{array}{l}\text { Up To Date Estética } \\
\text { Aplicada }\end{array}$ & $\begin{array}{l}\text { Revisão de } \\
\text { literatura. }\end{array}$ \\
\hline 19 & $\begin{array}{ll}\text { RIBEIRO } & \text { Ó, } \\
\text { CONSTANÇA }\end{array}$ & 2011 & $\begin{array}{l}\text { Manual de envelhecimento. } \\
\text { Lisboa }\end{array}$ & $\begin{array}{l}\text { Lidel Edições técnica, } \\
\text { Lda }\end{array}$ & Livro \\
\hline 20 & $\begin{array}{ll}\text { ROSS } & M H, \\
\text { PAWLINA W. } & \end{array}$ & 2012 & $\begin{array}{l}\text { Histologia - Texto e atlas: em } \\
\text { correlação com a biologia } \\
\text { celular e molecular. }\end{array}$ & $\begin{array}{l}\text { Guanabara Koogan/ } \\
\text { Rio de Janeiro }\end{array}$ & Livro \\
\hline 21 & STRUTZEL E et al & 2007 & $\begin{array}{l}\text { Análise dos fatores de riscos } \\
\text { para o envelhecimento da pele: } \\
\text { aspectos gerais e nutricionais }\end{array}$ & $\begin{array}{l}\text { Revista brasileira de } \\
\text { nutrologia clínica. }\end{array}$ & $\begin{array}{l}\text { Revisão de } \\
\text { literatura }\end{array}$ \\
\hline
\end{tabular}




\begin{tabular}{|l|l|l|l|l|l|}
\hline 22 & WHO & 2005 & $\begin{array}{l}\text { Envelhecimento ativo: uma } \\
\text { política de saúde }\end{array}$ & $\begin{array}{l}\text { OMS/ Organização } \\
\text { Pan-Americana da } \\
\text { Saúde }\end{array}$ & Livro \\
\hline
\end{tabular}

\section{Discussão}

\section{Envelhecimento}

O envelhecimento foi uma das temáticas mais discutida nas publicações e pode ser caracterizado como um processo biológico normal, que ocorre devido a processos fisiológicos realizados por nossas células (especialmente nas mitocôndrias). Nessa estrutura, através do metabolismo oxidativo, é gerada energia para as atividades vitais, como consequência 2 a $5 \%$ do oxigênio consumido gera Espécies Reativas de Oxigênio, sendo, algumas delas, radicais livres. Os radicais livres são moléculas altamente reativas que se não neutralizadas pelo sistema antioxidante desencadeia a lipoperoxidação de moléculas orgânicas, gerando lesões e acelerando $O$ envelhecimento (MARRONI; MORGAN-MARTINS; PORAWASKI, 2012).

O processo de envelhecimento é desencadeado pela divisão celular desordenada. Ao longo da vida, algumas células se multiplicam constantemente, a cada divisão, fragmentos de DNA são perdidos, causando pequenos erros genéticos que são passados para as células-filhas. Isso acontece até que a célula não consegue mais se dividir ou é destruída pelo próprio organismo por conter muitos erros, é exatamente isso que faz a vida chegar ao final. Porque esse envelhecimento dos órgãos, acarreta modificações que levam à diminuição da eficiência do seu funcionamento (ROSS e PAWLINA, 2007).

Frente a essa modificação do organismo muitas alterações na composição orgânica ocorrem, e consequentemente irão promover declínios em vários aspectos relacionados às habilidades e funções do corpo humano, citamos algumas: aumento da gordura corporal, redução da massa muscular magra e mudança no metabolismo de hormônios, redução no gasto energético diário e no percentual de água corporal. Esse fato resultará na redução do volume de alguns órgãos (RIBEIRO; CONSTANÇA, 2011).

O processo de envelhecimento, além dos aspectos que englobam a biologia e a genética, também sofre influência do estilo de vida que impõe condicionantes e determinantes que contribuirão positiva ou negativamente para - envelhecimento que cada indivíduo (STRUTZEL, 2007). Assim, o envelhecimento traz à tona a fragilidade da condição humana encarnando-se o "irredutível do corpo" por não ser mais capaz de participar do campo simbólico da sociedade. Mas, sim de um cenário dramático da velhice associada à pobreza, ao abandono, a doença, a dependência e aos múltiplos preconceitos que reforçam as suas incapacidades e perdas (LE BRETON, 2012).

No início do século XXI observa-se profundas transformações na estrutura populacional em vários países, inclusive no Brasil, como resultado de melhorias sociais e políticas de incorporações das novas tecnologias. O envelhecimento populacional neste século é acompanhado de ações amplas para criar políticas públicas que promovam estilo de vida saudável em todas as etapas da vida, favorecendo a prática de atividade física, o bem-estar geral, o lazer, o acesso a 
alimentos saudáveis e a redução do consumo de tabaco e álcool. Trazendo o conceito de envelhecimento ativo (OMS, 2005; BRASIL, 2014).

\section{Fatores que aceleram o processo de envelhecimento}

À medida que os indivíduos envelhecem as doenças crônicas nãotransmissíveis (DCNTs) tornam-se mais frequentes e podem desencadear incapacidades ou mesmo serem o fator que leva à mortalidade. Em muitas situações mais de uma doença crônica ocorre simultaneamente deixando o idoso mais vulnerável para desfechos negativos de morbimortalidade. As principais doenças crônicas que afetam os idosos no mundo são: doenças cardiovasculares (doença coronariana), hipertensão arterial sistêmica, acidente vascular cerebral (AVC), diabetes, câncer, doença pulmonar obstrutiva crônica, doença musculoesqueléticas, doenças mentais (principalmente demências e depressão), cegueira e diminuição da visão (OMS, 2005; BAUMAN, 2011).

Um dos fatores que desencadeiam as DCNTs ao longo do processo de envelhecimento está associado ao aumento do tecido gorduroso e redução da massa magra. O tecido adiposo acaba se depositando entre as vísceras contribuindo para o desenvolvimento das DCNTs, como a hipertensão e o diabetes levando à comorbidades como o Acidente Vascular Encefálico (AVE) (FRIES e PEREIRA, 2011). Portanto, a ingestão em excesso de alimentos pode provocar obesidade e com ela está associado as doenças crônicas. Por isso uma alimentação adequada é fundamental para um envelhecimento saudável (BRASIL, 2006). Para Ribeiro e Constança (2011) a alimentação não balanceada, exposição ao sol, o consumo de álcool e tabaco são consideradas medidas que dependendo da forma como são consumidas podem contribuir negativamente para saúde e desenvolvimento das DCNTs acelerando o processo de envelhecimento.

Além da obesidade o consumo excessivo de álcool é um dos fatores de risco com maior impacto na sociedade contemporânea, contribui de maneira significativa para ocorrência de DCNTs. A resistência ao consumo álcool se modifica ao longo da vida e, os idosos têm menor tolerância, assim o consumo em excesso pode comprometer a saúde (SÉ, 2009). Então o consumo de álcool pelos longevos é preocupante, por causar perda de independência nas atividades de vida diárias, tais como, diminuição do funcionamento intelectual, acidentes, episódios de confusão recorrentes, sintomas de autonegligência, diminuição da atenção e de atividades (NUNES, 2009).

Conforme Strutzel (2007) o consumo de tabaco é prejudicial em qualquer idade. Fumar além de acelerar o envelhecimento, faz com que o fumante perca em média dez anos de expectativa de vida, aumenta o risco de aparecimento de doenças, principalmente o câncer, problemas respiratórios e cardiovasculares, portanto as DCNTs (PORCELLO-MARRONE et al., 2013).

Outro fator que acelera o processo de envelhecimento é a exposição ao estresse prolongado levando a um desequilíbrio que desencadeia um aumento na produção de radicais livres, intensificando o processo de oxidação das células. Esse fenômeno é conhecido como estresse oxidativo, desempenha papel central na condução de eventos que causam o processo de envelhecimento. $O$ estresse oxidativo altera os ciclos de renovação celular e provocando danos ao DNA, aumentando a produção de substâncias inflamatórias. Esse desequilíbrio leva a reações em cascata que vão desde o 
aumento dos níveis de colesterol ruim, deposição de gordura no fígado, diabetes tipo 2, elevação da pressão arterial, do risco de aterosclerose e consequentemente de doenças cardíacas e cerebrovasculares (FRIES; PEREIRA, 2011; MARRONI, MORGAN-MARTINS e PORAWASKI, 2012).

\section{Fatores que retardam o processo de envelhecimento}

Pela OMS (2005), fatores que retardam o envelhecimento diz respeito ao envelhecimento ativo e envolve um indivíduo ou grupo populacional. Envelhecimento ativo é perceber o potencial para o bem-estar físico, social e mental ao longo do curso da vida, para que os indivíduos possam participar da sociedade de acordo com suas necessidades, desejos e capacidades e ao mesmo tempo propiciar proteção, segurança e cuidados adequados, quando necessários.

Envelhecer bem, diz respeito a ter saúde, educação continuada, participação ativa nos avanços sociais e econômicos, bem como, um sistema de proteção social para os mais pobres e excluídos, sendo prioridades explícitas das políticas sociais e de segurança. Para envelhecer bem são necessários: autoeficácia; autoestima; otimismo e bom humor para que se possa planejar a vida e a morte. Bem como, é necessário saúde e oportunidades de estudo sempre que se desejar, bem como, poder relacionar-se socialmente, ter oportunidade de trabalho digno e um suporte financeiro, porque o envelhecer traz consigo alterações morfofisiológicas que requerem maior atenção e cuidado (KALACHE A, 2008; BEARD et al., 2012).

Portanto, para envelhecer bem são necessários o autocuidado para que o idoso possa gerir suas atividades de vida diária e, para isso o suporte familiar é essencial. Assim a presença de uma rede de apoio, a família e a comunidade onde está inserido, tem um papel fundamental no autocuidado e, por fim, o cuidado institucional, onde o estado aplique as normativas já existentes no estatuto do idoso (BEARD et al; 2012)

Os determinantes que favorecem a preservação do organismo por mais tempo estão associados ao estilo de vida saudável, porque envelhecer é um processo. Portanto, é essencial que esses hábitos estejam presentes ao longo de todo o ciclo de vida que compreendem: ausência de consumo de tabaco e álcool; a atividade física regular; aprendizado permanente; alimentação saudável; saúde bucal e a gestão do regime medicamentoso (CAMPOS; NETO, 2008).

De acordo com Ferreira (2009) para uma longevidade saudável e uma expectativa de vida com qualidade é necessário que o indivíduo mantenha seu potencial de bem-estar físico, social e mental, através de uma a participação ativa nas questões econômicas, culturais, espirituais e cívicas, evitar comportamentos de riscos são atitudes que contribuirão para um processo do envelhecimento saudável (RIBEIRO; CONSTANÇA, 2011).

Os cuidados para intervir e subsidiar os cidadãos na manutenção da inteligência, habilidade verbal durante as fases da vida, conciliada a combinação de informação cognitiva e educacional, são eventos relacionados à diminuição dos problemas de saúde física, mental e social, ocasionam uma longevidade com saúde (MINAYO, ASSIS, 2017). 
A atividade física, se for regular, pode trazer grandes benefícios à saúde de todos os indivíduos, além de retardar o declínio funcional e consequentemente o envelhecimento. As atividades corporais são consideradas um dos hábitos mais valiosos para as pessoas alcançarem um envelhecimento bem-sucedido. Assim, é necessário lembrar que o conceito de atividade vai além dos exercícios físicos, faz parte desse conceito a estimulação das capacidades cognitivas, que são atitudes que contribuem para um declínio mais tardio das funções intelectuais (SÉ, 2009).

Em um estudo realizado em uma universidade que investigou 71 idosas com idade de $69,7 \pm 5,8$ anos, peso corporal de $64,7 \pm 11,7 \mathrm{~kg}$ e estatura de $1,60 \pm 0,10 \mathrm{~m}$, com queixa de dores musculoesquelética. Ao iniciarem a pratica atividade física, pelo menos uma vez por semana, reduziu significativamente as dores e melhorou muito a qualidade de vida (PEREIRA et al., 2009).

Segundo Freitas; Queiroz; Sousa (2010) outra atitude importante na preservação da autonomia e independência da pessoa é manter os seus próprios cuidados em todas as fases do ciclo vital. Reforçando que a educação permanente e uma vida sexual ativa, são comportamentos que podem prevenir doenças e prolongar a vida com mais saúde e prazer.

\section{Longevidade e estilo de vida}

De acordo com a OMS, 2006 a maioria dos países classifica a terceira idade a partir dos 60 anos, considerando o ponto de vista econômico, visto que o pagamento da aposentadoria começa aos 60 anos para as mulheres e aos 65 para os homens. Essa classificação coincide com o conceito da ONU que estabelece que a Terceira Idade comece aos 60 anos nos países subdesenvolvidos e 65 anos nos países desenvolvidos.

Mediante todas as dimensões que envolvem o envelhecimento, o termo longevidade não está contido em um único conceito, considerando que a idade cronológica pode não ser idêntica à idade biológica, psicológica e social do indivíduo (ALMEIDA; GUTIERREZ; MARQUES, 2012). Portanto, o conceito de longevidade será naturalmente a superação da velhice, uma consequência do envelhecimento ativo e produtivo que, propõe um convite ao idoso a um estilo de vida para manter as dimensões biológica, psicológica, social e financeira mais saudável possível (FERREIRA, 2009).

No entanto, a proposta dessa longevidade só acontecerá quando o mesmo for capaz de continuar assumindo suas responsabilidades, construindo sua história com independência, autonomia, esperança e felicidade, garantindo manutenção pessoal, direitos e respeito com dignidade. Para mensurar diretamente a saúde das pessoas, têm-se desenvolvido e aprovado instrumentos estruturados e simplificados, capazes de reconhecer os estados de "completo bem-estar físico, mental e social", a mensuração da Qualidade de Vida (QV) é algo que terá muita importância no século $\mathrm{XXI}$, tanto nas práticas assistenciais quanto nas políticas públicas, nos campos de prevenção de doenças e promoção da saúde (PS) (CAMPOS; NETO, 2008).

De acordo com Ministério da Saúde do Brasil (2014), são atividades prioritárias para promoção da saúde: formação e educação permanente, alimentação adequada e saudável, práticas corporais e atividades físicas, enfrentamento do uso do tabaco e seus derivados, enfrentamento do uso 
abusivo de álcool e outras drogas. Portanto, criar hábitos de vida saudáveis é essencial para promover uma longevidade com saúde.

A longevidade é uma realidade contemporânea e para manter a saúde dos longevos, sem dúvida, a qualidade de vida estará diretamente associada promoção da saúde que consequentemente resultará em saúde, independência e autonomia (FERREIRA, 2009). Um conceito ampliado e positivo de qualidade de vida tem como foco o processo social de sua produção. Elege como objetivo a defesa da vida e o desenvolvimento humano. Superando o modelo de intervenção e, passando para práticas intersetoriais e conhecimentos interdisciplinares, que influenciam na vida das pessoas estabelecendo uma maior corresponsabilidade pela sua saúde (IRIGARAY; TRENTINI, 2009).

\section{Promoção da saúde}

De acordo com a Organização Mundial da Saúde (2006), a PS é a completa condição humana de bem-estar físico, mental e social, essa condição segunda a Lei Federal Brasileira № 8.080/1990 tem como determinantes e condicionantes, entre outros, a alimentação, a moradia, o saneamento básico, o meio ambiente, o trabalho, a renda, a educação, a atividade física, o transporte, o lazer e o acesso aos bens e serviços essenciais.

No campo da saúde pública a PS vem ganhando destaque. A visão entre condições sociais e saúde aprofunda a discussão que apresenta a PS como a estratégia central para garantir a qualidade de vida do cidadão. Apresenta-se como fator principal para diminuir o risco de doenças e aumentar as chances de longevidade com saúde, o que acarreta em uma intervenção multi e intersetorial sobre os chamados determinantes do processo de envelhecimento (RIBEIRO; CONSTANÇA 2011).

Entre os especialistas no assunto ainda não existe um consenso sobre o que constitui qualidade de vida, mas há uma tentativa de definição que engloba o estado de saúde geral. Bem como, uma variedade de domínios, mas é considerado como fatores determinantes e condicionantes a educação, meioambiente, recursos econômicos, relacionamentos, trabalho, alimentação, atividade física, meditação e lazer (BRYANT; ALPETER; WHITELAW, 2006).

O Brasil (2014) preconiza a PS como um mecanismo de fortalecimento e implantação de uma política transversal, integrada e intersetorial. Onde se possa estabelecer o diálogo entre as diversas áreas e a sociedade para compor uma rede de compromisso e corresponsabilidade com a Qualidade de Vida da população.

\section{Conclusão}

O presente artigo trouxe reflexões acerca do envelhecimento, fatores que retardam e que aceleram o envelhecimento, longevidade e estilo de vida e promoção da saúde.

A longevidade é o envelhecimento prolongado, e se requer que ela seja saudável, a partir da aquisição de bons hábitos ao longo da vida, uma vez que envelhecer é um processo. Construir um envelhecimento saudável vai muito além de hábitos isolados, passa por uma política de PS, focada em promover o envelhecimento saudável, com a prática de atividade física, lazer e bem-estar. 
Envelhecimento ativo e com saúde está relacionado ao potencial para o bem-estar físico, social e mental ao longo do curso da vida, para que os idosos possam participar da sociedade de acordo com suas necessidades, desejos e capacidades e ao mesmo tempo que a sociedade possa propiciar proteção, segurança e cuidados adequados, quando necessários.

\section{Referências}

ALMEIDA, M; GUTIERREZ, G; MARQUES, R. Qualidade de vida. São Paulo: Edições EACH. 2012. [Consult. 13 abr. 2019]. Disponível em: http://each.uspnet.usp.br/edicoes-each/qualidade vida.pdf

BAUMAN, Z. Diálogos com Zygmunt Bauman. Entrevista para a CPFL Cultura e o Seminário Fronteiras do Pensamento. 2011. Disponível em: https://www.institutocpfl.org.br/2011/08/16/dialogos-com-zygmunt-bauman/

BEARD, J. et al. Global Population Ageing: Peril or Promise? PGDA Working Paper No. 89. disponível em: http://www.hsph.harvard.edu/pgda/working.htm.

BRASIL, Ministério da Saúde. Portaria no 2.448. de 11 de novembro de 2014. Redefine a Política Nacional de Promoção da Saúde. Disponível em: https://bvsms.saude.gov.br/bvs/saudelegis/gm/2014/prt2446 $11 \quad 11$ 2014.html

BRASIL, Presidência da República, Casa Civil, Subchefia para Assuntos Jurídicos. Lei N. 8.080, de 19 de setembro de 1990 (Legislação do SUS). Disponível em: http://www.planalto.gov.br

BRASIL, Ministério da Saúde. Programa Nacional para a Saúde das Pessoas Idosas. Brasília; 2006.

BRYANT L, ALPETER M, WHITELAW N. Evaluation of health promotion programs for older adults: An introduction. Journal of Applied Gerontology. [Em linha]. June 2006, vol 25, no 3 [Consult. 16 junho. 2019]

CAMPOS M, NETO J. Qualidade de vida: um instrumento para promoção da saúde. Revista Baiana de Saúde Pública. [Em linha].2008, vol 32, no 2 [Consult. 3 julho. 2018], pp. 232-240. Disponível em:http://rbsp.sesab.ba.gov.br/index.php/rbsp/article/view/1438

COSTA, JJS A educação segundo Paulo Freire: uma primeira análise filosófica. Theoria - Revista Eletrônica de Filosofia Faculdade Católica de Porto Alegre. Volume VII - Número 18 - Ano 2015 - ISSN 1984-9052. P. 76. http://www.theoria.com.br/edicao18/06182015RT.pdf 
FERNÁNDEZ, BR. Envejececimiento activo. Contribuciones de la Psicología. [Em linha]. Madrid 2009, no13 [Consult. 24 de agosto. 2018], pp.214216. Disponível em: https://www.aidep.org/sites/default/files/201707/RIDEP\%2030\%20-com2.pdf

FERREIRA, A. A Qualidade de Vida em Idosos em Diferentes Contextos Habitacionais: A Perspectiva do Próprio e do Seu Cuidador. Dissertação de Mestrado, Faculdade de Psicologia e Ciências da Educação, Universidade de Lisboa, 2009.

FONSECA A. O envelhecimento. Uma abordagem psicológica. [Em linha]. Lisboa: Universidade Católica Editora, 2006. [Consult. 5 fev. 2019]. Disponível em:

https://www.uceditora.ucp.pt/site/custom/template/ucptpl uce.asp?SSPAGEID= 1295\&lang=1\&artigolD $=373$

FREITAS, M; QUEIROZ, T; SOUSA, J. O significado da velhice e da experiência de envelhecer para os idosos. Revista da Escola de Enfermagem da Universidade de São Paulo, 44(2), 407-412. Recuperado em 02 março, 2019, disponível em: https://doi.org/10.1590/S0080-62342010000200024

FRIES, AT; PEREIRA, DC. Teorias do envelhecimento. Revista Contexto \& Saúde, ljuí • v. $10 \cdot$ n. $20 \cdot$ Jan./jun. 2011.

GIACOMIN, KC; SANTOS, WJ; FIRMO, JOA. O luto antecipado diante da consciência da finitude: a vida entre os medos de não dar conta, de dar trabalho e de morrer. Ciênc. Saúde Coletiva [online]. 2013, vol.18, n.9, pp.2487-2496. ISSN 1413-8123. Disponível em: https://doi.org/10.1590/S141381232013000900002 .

GOVERNO DE PORTUGAL. Ano Europeu do Envelhecimento Ativo e da Solidariedade entre Gerações programa de ação. [Em linha]. Portugal, 2012 [Consult. 18 abr. 2019]. Disponível em: https://www.sns.gov.pt/wpcontent/uploads/2017/07/ENEAS.pdf

IRIGARAY, T; TRENTINI C. Qualidade de vida em idosas: a importância da dimensão subjetiva. Estudos de Psicologia. [Em linha]. Julho/ setembro, 2009, vol. 23, no3 [Consult. 23 agosto. 2019], pp.297-304. Disponível em: http://www.scielo.br/pdf/estpsi/v26n3/v26n3a03.pdf.

LLOYD-SHERLOCK, P. et al. Population ageing and health. Lancet. $2012 \mathrm{Apr}$ 7;379(9823):1295-6. 
LE BRETON D. Sociologia do corpo. editora: vozes, 6 ed. são Paulo, 2012.

KALACHE, A. O mundo envelhece: é imperativo criar um pacto de solidariedade. Social Ciência \& Saúde Coletiva, 13(4):1107-1111, 2008.

MINAYO, MCS; ASSIS, SG. Novas e velhas faces da violência no Século XXI: Visão da literatura brasileira do campo da saúde. Rio de Janeiro, Editora Fiocruz, 2017. p.323-340.

MARRONI, NP; MORGAN-MARTINS, MI; PORAWASKI, M. Radicais livres no processo saúde-doença: da bancada à clínica. Curitiba, PR: CRV, 2012. ISBN 978-85-8042-424-9

NUNES, IM. A Constelação do Idoso. Artigo de Investigação Médica. [Em linha]. Instituto de Ciências Biomédicas Abel Salazar, 2009. Mestrado Integrado em Medicina [Consult. 5 agosto. 2019]. Disponível na Internet: http://repositorioaberto.up.pt/bitstream/10216/21066/2/a\%20constela0\%20do\%20idoso.pdf.

ORGANIZAÇÃO MUNDIAL DE SAÚDE (OMS) - Envelhecimento ativo. Um Projeto de Política de Saúde: [em linha]. Madrid: OMS, 2006. [Consult. 5 agosto de 2019]. Disponível na Internet: URL:http://www.crdeunati.uerj.br/doc gov/destaque/Madri.doc.

PEREIRA, ÉF; TEIXEIRA, CS; DARONCO, LSE. Estilo de vida, prática de exercício físico e dores musculoesqueléticas em idosas fisicamente ativas. RBCEH, Passo Fundo, v. 6, n. 3, p. 343-352, set/dez. 2009).

PORCELLO-MARRONE, LC. et al. "Risk factors among stroke subtypes in Brazil," Journal of Stroke and Cerebrovascular Diseases, vol. 22, no. 1, pp. 3235, 2013.)

RESENDE, MR. Imunocosmética estimulação de células imunocompetentes para a proteção da pele e reparação do DNA. Up To Date Estética Aplicada, v 15. N. 71, p. 20-22, jan. 2009.

RIBEIRO, Ó; CONSTANÇA, P. Manual de envelhecimento. Lisboa: Lidel Edições técnica, Lda, 2011. ISBN 978-972-75-77-392.

ROSS MH, PAWLINA W. Histologia - Texto e atlas: em correlação com a biologia celular e molecular, 6 ed. Rio de Janeiro: Guanabara Koogan, 2012. 987p. 
SÉ, E. Caminhos para uma velhice bem-sucedida. [Em linha]. 2009, [Consult. 5 set. 2019], Disponível em: http://www2.uol.com.br/vyaestelar/velhice bem sucedida.htm.

SILVA EL, MENEZES EM. Metodologia da pesquisa e elaboração de dissertação. Florianópolis: UFSC, 2001.

STRUTZEL, E; CABELLO, H; QUEIROZ, L; FALCÃO, MC. Análise dos fatores de riscos para o envelhecimento da pele: aspectos gerais e nutricionais. Rev Bras Nutr Clin. São Paulo. V.22, n.2, p. 139-145, 2007.

UNITED NATIONS, Department of Economic and Social Affairs, Population Division (2019). World Population Prospects 2019, Volume II: Demographic Profiles (ST/ESA/SER.A/427)

WHO - Envelhecimento ativo: uma política de saúde / World Health Organization; tradução Suzana Gontijo. - Brasília: Organização Pan-Americana da Saúde, 2005. 60p.: il. Disponível em: http://bvsms.saude.gov.br/bvs/publicacoes/envelhecimento ativo.pdf 